\title{
Platelet-to-Lymphocyte and Neutrophil-to-Lymphocyte Ratio as Predictive Biomarkers for Early-onset Neonatal Sepsis
}

\author{
Shujian Zhang ${ }^{1}$, Xue Luan ${ }^{2}$, Wei Zhang ${ }^{3}$ and Zhengyong Jin ${ }^{1}$ \\ ${ }^{1}$ Department of Pediatrics, Affiliated Hospital of Yanbian University, Jilin, China \\ ${ }^{2}$ Department of Pediatrics, First Hospital, Jilin University, Jilin, China \\ ${ }^{3}$ Health Management Center, Children's Hospital of Changchun City, Jilin, China
}

\begin{abstract}
Objective: To determine the predictive significance of platelet-to-lymphocyte ratio (PLR) and neutrophil-to-lymphocyte ratio (NLR) in early-onset neonatal sepsis (EONS).

Study Design: A descriptive study.

Place and Duration of Study: The Neonatal Intensive Care Unit (NICU), Affiliated Hospital of Yanbian University, Jilin, China, from January 2018 to January 2020.

Methodology: Of the total 124 children, 74 children with EONS were enrolled in group A and 50 children without infection-related diseases were enrolled in group B (control). The EONS risk factors were evaluated by logistic regression. Besides, the PLR and NLR diagnostic performances in EONS were evaluated by plotting the receiving operating characteristic (ROC) curves.

Results: In the univariate analysis, the differences for platelet count, lymphocyte number, neutrophil number, NLR, and PLR, between group $A$ and group $B$ were of statistical significance $(p=0.02,0.021,<0.001,<0.001$, and $<0.001$ respectively). As suggested by logistic regression, PLR and NLR were identified as the factors to independently predict the risk of EONS ( $p=$ 0.012 , and 0.003 , respectively). In addition, the value of area under the ROC curve (AUC) of NLR in predicting EONS was 0.788 ( $95 \% \mathrm{Cl}: 0.708-0.868 ; p<0.001$ ), which was greater than that of PLR. At the NLR value of $\geq 3.169$, the sensitivity of predicting EONS was $77 \%$, and the specificity was $78 \%$.

Conclusion: Peripheral blood NLR and PLR have high predictive value for EONS. The predictive value of NLR as a biomarker for EONS evaluation was greater than that of PLR.
\end{abstract}

Key Words: Neonatal sepsis, Logistic models, ROC curve, Blood cell count.

How to cite this article: Zhang S, Luan X, Zhang W, Jin Z. Platelet-to-Lymphocyte and Neutrophil-to-Lymphocyte Ratio as Predictive Biomarkers for Early-onset Neonatal Sepsis. J Coll Physicians Surg Pak 2021; 31(07):821-824.

\section{INTRODUCTION}

Neonatal sepsis is the third leading cause of neonatal death after a premature delivery and related complications during delivery. ${ }^{1}$ Approximately 400,000 newborns die every year globally due to neonatal sepsis. ${ }^{2}$ In developing countries, due to a limited level of early diagnosis and treatment, the mortality rate by neonatal sepsis is about three times that of the developed countries. ${ }^{3}$ According to the time of onset, neonatal sepsis can be divided into early-onset (onset within $72 \mathrm{~h}$ after birth) and late-onset (onset $>72 \mathrm{~h}$ after birth). ${ }^{4,5}$ The occurrence of EONS is related to several perinatal factors, such as retrograde infection of pathogenic bacteria through the placenta or vagina. ${ }^{6}$

Correspondence to: Dr. Zhengyong Jin, Department of Pediatrics, Affiliated Hospital of Yanbian University, Jilin, China

E-mail: zhengyongjin1958@163.com

Received: March 22, 2021; Revised: May 09, 2021; Accepted: June 01, 2021

DOI: https://doi.org/10.29271/jcpsp.2021.07.821
The results of blood culture, currently the gold standard for diagnosing sepsis, can take a minimum of $48 \mathrm{~h}$. The sensitivity of such blood culture is low and is closely associated with the amount of blood sample, the location of collecting blood samples, the use of antibiotics, and the level of contamination of the blood sample. ${ }^{7}$ As the defence barrier function and immune system development of neonates remain incompletely developed and the condition of neonatal sepsis progresses rapidly, early diagnosis and accurate treatmentare the keys to reducing mortality.

In recent years, NLR and PLR indicators derived from blood analysis have attracted attention in the research of inflammation-related diseases. According to some studies, NLR and PLR can be used as prognostic indicators for cancer and cardiovascular diseases ${ }^{8,9} \mathrm{NLR}$ indicates the ratio of neutrophils to lymphocytes. Studies on sepsis in adults showed that NLR could be used as a biomarker for evaluating systemic inflammation. ${ }^{10}$ PLR implies the ratio of platelets to lymphocytes. A study showed PLR to be a useful marker for evaluating patients' inflammatory response and disease activity. ${ }^{9}$ 
The current studies on NLR and PLR are mostly related to adult diseases, and reports on EONS are scarce. Therefore, the aim of this study was to examine the predictive value of NLR and PLR in EONS evaluation.

\section{METHODOLOGY}

The Ethics Committee of the Hospital approved this descriptive study. Seventy-four children with EONS admitted to the Affiliated Hospital of Yanbian University, Jilin, China, from January 2018 to January 2020, were classified as group A. Forty-five children without any infectious diseases were taken in group $B$.

The inclusion criteria were positive blood culture within three days after birth, accompanied by clinical symptoms of systemic infection (unstable body temperature, hyperthermia, or hypothermia; unstable heartbeat rhythm, tachycardia or bradycardia; irregular breathing rhythm regularity, shortness of breath or pauses; feeding intolerance, etc. ). No antibiotic treatment was given before the blood sampling. Prior informed consent of the parents of each child was obtained. The patients medical history were complete. Exclusion criteria were: mother drinking and/or smoking during pregnancy; mother taking medicines that affect the fetal hematopoietic system during pregnancy; and mother with chorioamnionitis, diabetes, or preeclampsia during pregnancy; mother having used steroids before delivery; twins or multiple pregnancies; premature babies (gestational age <37 weeks); post-term babies (gestational age $>42$ weeks); children less than or greater than gestational age; and children receiving cardiopulmonary resuscitation after delivery.

Gender, birth mode, birth weight, gestational age, neutrophil count, lymphocyte count, platelet count, NLR, and PLR of children were duly recorded in both groups. Blood samples were collected on admission for blood analysis and blood culture. After collecting blood samples, antibiotics were used prophylactically until the results of blood culture and drug susceptibility were obtained. Statistical software, SPSS version 25.0, was employed for data analysis. The measurement data were represented by the mean and standard deviation and median (IQR: $25^{\text {th }}$ percentile- $75^{\text {th }}$ percentile). For data with normal distribution or approximately normal distribution, the independent ttest ( $\mathrm{t}$ ) was used to compare the two groups. For comparing data with non-normal distribution in both groups, Mann-Whitney Utest was used. Sample size and percentages were used to represent enumerated data. Chi-square test $\left(c^{2}\right)$ was used for comparison between two groups. A logistic regression model was used to determine the influence of factors such as NLR and PLR on EONS. The area under the receiver operating characteristic (ROC) curve (AUC) was used to determine the sensitivity and specificity of both NLR and PLR for predicting EONS. Statistical significance was defined as $p$-value $<0.05$.

\section{RESULTS}

Univariate analysis showed that in terms of platelet count, lymphocyte number, neutrophil number, NLR, and PLR, differences between group $A$ and group $B$ were of statistical signifi- cance ( $p<0.001$ or $p<0.05$, respectively, Table I). However, no statistically significant difference in gender, gestational age, production style, and birth weight ( $p=0.234,0.492,0.144$, and 0.862 , respectively) was noticed (Tablel).

Logistic regression analyses on lymphocyte number, neutrophil number, NLR, and PLR, were performed. The results indicated that both NLR and PLR are independent risk factors for EONS with a statistical significance $(p<0.05$, Table II).

Table I: Basic situation of the two groups of children.

\begin{tabular}{|l|c|c|c|}
\hline Index & Group A (n= 74) & Group B (n= 50) & p-value \\
\hline Gender [male, $\mathrm{n}(\%)]$ & $45(60.8)$ & $25(50)$ & 0.234 \\
\hline Gestational week & $38(35-40)$ & $38(37-9)$ & 0.492 \\
\hline $\begin{array}{l}\text { Delivery route } \\
\text { [NSD, } \mathrm{n}(\%)]\end{array}$ & $36(48.6)$ & $18(36)$ & 0.144 \\
\hline Birth weight(kg) & $3.17(2.39-3.54)$ & $3.06(2.88-3.45)$ & 0.862 \\
\hline Lymphocyte $\left(10^{9} / \mathrm{L}\right)$ & $2.64(2.04-3.62)$ & $3.16(2.48-4.07)$ & 0.021 \\
\hline Neutrophil $\left(10^{9} / \mathrm{L}\right)$ & $10.1(7.24-14.58)$ & $7.78(6.04-9.89)$ & $<0.001$ \\
\hline PLT $\left(10^{9} / \mathrm{L}\right)$ & $255.24 \pm 68.61$ & $231.36 \pm 44.26$ & 0.02 \\
\hline NLR & $4.16(3.26-5.61)$ & $2.35(1.97-3.16)$ & $<0.001$ \\
\hline PLR & $99.57(69.61-128.57)$ & $71.53(53.98-88.29)$ & $<0.001$ \\
\hline
\end{tabular}

Table II: Multivariate logistic regression analysis of early-onset neonatal sepsis.

\begin{tabular}{|l|c|c|c|}
\hline Risk factor & B & P-value & OR (95\%C.I) \\
\hline Lymphocyte & 0.715 & 0.068 & $2.043(0.948-4.404)$ \\
\hline Neutrophil & 0.057 & 0.588 & $1.058(0.863-1.298)$ \\
\hline NLR & 2.753 & 0.003 & $15.697(2.573-95.747)$ \\
\hline PLR & 2.446 & 0.012 & $11.540(1.716-77.595)$ \\
\hline
\end{tabular}

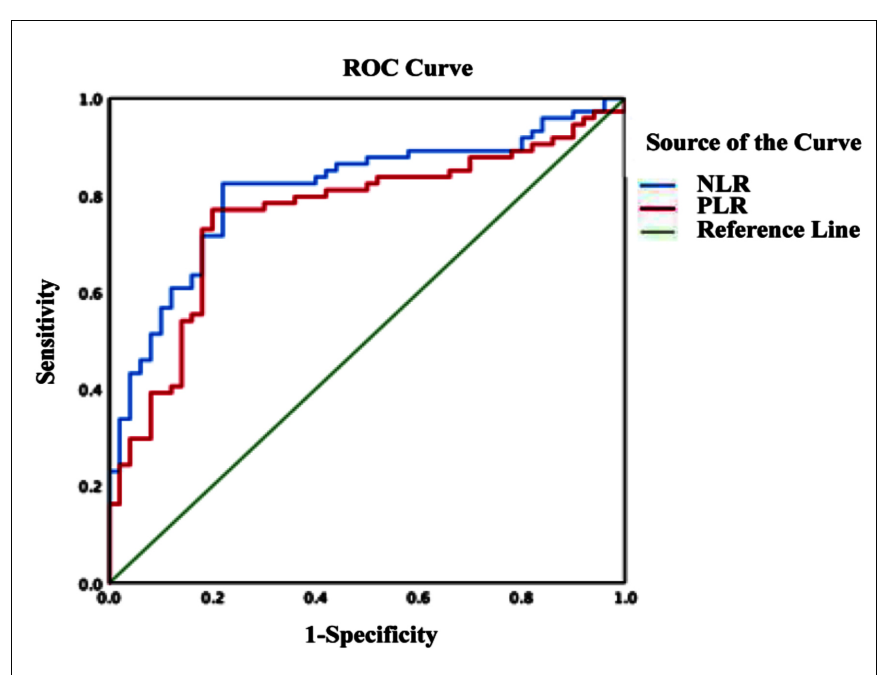

Figure 1: ROC curves of NLR and PLR predict early-onset neonatal sepsis.

The ROC area of NLR predicted for EONS was $0.788(95 \% \mathrm{Cl}$ : $0.708-0.868, p<0.001)$. The best cutoff value of NLR affecting EOS was 3.169. Its sensitivity for predicting EONS was $77 \%$, and specificity was $78 \%$, the positive predictive value was $85 \%$, and the negative predictive value was $75 \%$.

The ROC area of PLR predicted for EONS was $0.699(95 \% \mathrm{Cl}$ : 0.607-0.792; $p<0.001$ ).

The best cutoff value of PLR affecting EOS was 90.846 . Its sensitivity for predicting EONS was $64.9 \%$, and specificity was $80 \%$, 
the positive predictive value was $79 \%$, and the negative predictive value was 76.9\% (Figure 1).

NLR and PLR can predict EONS. The ROC area of NLR predicting EONS was 0.788 , which was higher than the ROC area of PLR.

\section{DISCUSSION}

Along the improvement in neonatal care, the mortality rate of neonatal sepsis has declined, butEONS remains one of the principal causes of neonatal morbidity and death. Early diagnosis and treatment may control the progress of systemic inflammatory response in time and reduce the mortality by sepsis. ${ }^{11}$ However, the clinical manifestations of neonatal sepsis are not specific, and the neonatal immunity is observed low after birth, and the disease progressions occur rapidly, making an early diagnosis of the disease more difficult. Therefore, there is an urgent need to find a rapid and specific biomarker for EONS.

Blood analysis involves a simple and easily available examination, under which neutrophil count, lymphocyte count, and platelet count are commonly used clinical indicators. ${ }^{12}$ When inflammation occurs, neutrophil innate immune regulation is an important link; ${ }^{13}$ neutrophil colony-stimulating factors can delay granulocyte apoptosis, thereby increasing the neutrophil counts. At the same time, several cytokines and chemokines inhibit the proliferation and activation of lymphocytes and reduce the number of lymphocytes throughout the body. ${ }^{14}$ Also, during systemic inflammation, megakaryocytes too, accelerate their proliferation rate, resulting in thrombocytosis. ${ }^{15}$ However, the predictive value of platelets in sepsis remains unclarified. Several studies have reported an association of the platelet count to the severity of the disease. ${ }^{16}$ Inflammation inhibits the bone marrow and reduces the number of platelets. Emrah et al. reported that platelets were not associated with EONS. ${ }^{17}$ This study showed that patients from both groups had a statistically significant difference in neutrophil count and lymphocyte count. Next, logistic regression analysis of the neutrophil count and lymphocyte count showed that these two markers are independent risk factors for EONS.

In recent years, the NLR and PLR biomarkers, derived from the analysis of peripheral blood, have drawn people's attention in the research of inflammation-related diseases. Both NLR and PLR can be derived from peripheral blood analysis. Compared with the individual neutrophil count, platelet count, and lymphocyte count, NLR and PLR are more accurate and stable. ${ }^{18}$ Previous studies ${ }^{19-21}$ have shown that sepsis in adults, pneumonia, cancer, and systemic inflammatory diseases, NLR and PLR become indicators to predict the severity and prognosis of the disease. This study highlighted statistically significant differences between group A and group B in terms of NLR and PLR. Furthermore, logistic regression analysis on NLR and PLR revealed that NLR and PLR are independent risk factors for EONS.

\section{CONCLUSION}

Overall, both NLR and PLR are independent risk factors for earlyonset neonatal sepsis. The predictive value of NLR and PLR for early-onset neonatal sepsis is higher, and the predictive value of NLR is higher than that of PLR.

\section{ETHICALAPPROVAL:}

This retrospective descriptive study, involving human participants, was in accordance with the ethical standards of the Institutional and National Research Committee; and with 1964 Helsinki Declaration and its later amendments or comparable ethical standards. The Ethics Committee of Yanbian University School of Medicine approved this study.

\section{PATIENTS' CONSENT:}

Informed consents were obtained from the patients to publish the data concerning this case.

\section{CONFLICT OF INTEREST:}

The authors declared no conflict of interest.

\section{AUTHORS' CONTRIBUTION:}

SZ: Design, literature search, data acquisition, data analysis, and manuscript preparation and editing.

$\mathrm{XL}$ : Drafting the work and manuscript preparation and editing. WZ: Revising it critically for important intellectual content. Z): Agreement to be accountable for all aspects of the work.

\section{REFERENCES}

1. Liu L, Johnson HL, Cousens S, Perin J, Scott S, Lawn JE, et al. Global, regional, and national causes of child mortality: An updated systematic analysis for 2010 with time trends since 2000. Lancet 2012; 379(9832):2151-61. doi: 10.1016/s0140-6736(12)60560-1.

2. Lawn JE, Cousens S, Zupan J. 4 million neonatal deaths: When? Where? Why? Lancet 2005; 365(9462):891-900. doi: 10.1016/s0140-6736(05)71048-5.

3. Khan F. C-reactive protein as a screening biomarker in neonatal sepsis. J Coll Physicians Surg Pak 2019; 29(10): 951-3.

4. Ershad M, Mostafa A, Cruz M, Vearrier D. Neonatal sepsis. Curr Emerg Hos Med Rep 2019; 7(3):83-90. doi: 10.1007/s40138-019-00188-z.

5. Odabasi IO, Bulbul A. Neonatal sepsis. Sisli Etfal Hastan Tip Bul 2020; 54(2):142-58. doi: 10.14744/semb.2020.00236.

6. Shane AL, Sánchez PJ, Stoll BJ. Neonatal sepsis. Lancet 2017; 390(10104): 1770-80. doi: 10.1016/s01406736(17)31002-4.

7. Iroh Tam PY, Bendel CM. Diagnostics for neonatal sepsis: Current approaches and future directions. Pediatr Res 2017; 82(4):574-83. doi: 10.1038/pr.2017.134.

8. Lalosevic MS, Markovic AP, Stankovic S, Stojkovic M, Dimitrijevic I, Vujacic IR, et al. Combined diagnostic efficacy of neutrophil-to-lymphocyte ratio $(\mathrm{n} / \mathrm{r})$, platelet-tolymphocyte ratio (PLR), and mean platelet volume (MPV) as biomarkers of systemic inflammation in the diagnosis of colorectal cancer. Disease Markers 2019; 2019:1-7. doi: $10.1155 / 2019 / 6036979$.

9. Qin B, Ning M, Tang Q, Wei T, Zhong R, Yang M, et al. Neutrophil to lymphocyte ratio (NLR) and platelet to lymphocyte ratio (PLR) were useful markers in assessment of inflammatory response and disease activity in SLE 
patients. Mod Rheumatol 2016; 26(3):372-6. doi: 10.3109/ 14397595.2015.1091136.

10. Dragan D, Goran R, Maja S, Ivan S, Ivo U, Tamara A, et al. Neutrophil-to-lymphocyte ratio, monocyte-to-lymphocyte ratio, platelet-to-lymphocyte ratio, and mean platelet volume-to-platelet count ratio as biomarkers in critically ill and injured patients: Which Ratio to choose to predict outcome and nature of bacter. Mediators Inflamm 2018; 2018:1-15. doi: 10.1155/2018/3758068.

11. Mukhopadhyay S, Taylor JA, Kohorn IV, Flaherman V, Burgos AE, Phillipi CA, et al. Variation in sepsis evaluation across a national network of nurseries. Pediatrics 2017; 139(3):e20162845. doi: 10.1542/peds.2016-2845.

12. Camacho-Gonzalez A, Spearman PW, Stoll BJ. Neonatal infectious diseases: Evaluation of neonatal sepsis. Pediatr Clin North Am 2013; 60(2):367-89. doi: 10.1016/j.pcl. 2012.12.003.

13. Kovach MA, Standiford TJ. The function of neutrophils in sepsis. Curr Opin Infec Dis 2012; 25(3):321-7. doi: 10.1097/ QCO.0b013e3283528c9b.

14. Neul-Bom Y, Choonhee S, Soo-Jung U. Role of the neutrophil-lymphocyte count ratio in the differential diagnosis between pulmonary tuberculosis and bacterial community-acquired pneumonia. Ann Lab Med 2013; 33(2):105-10. doi: 10.3343/alm.2013.33.2.105.

15. Allen E, Bakke AC, Purtzer MZ, Deodhar A. Neutrophil CD64 expression: Distinguishing acute inflammatory autoimmune disease from systemic infections. Ann Rheum Dis 2002; 61(6):522-5. doi: 10.1136/ard.61.6.522.
16. Hudzik B, Szkodzinski J, Gorol J, Niedziela J, Lekston A, Gasior M, et al. Platelet-to-lymphocyte ratio is a marker of poor prognosis in patients with diabetes mellitus and STelevation myocardial infarction. Biomark Med 2015; 9(3):199-207. doi: 10.2217/bmm.14.100.

17. Can E, Hamilcikan Ş, Can C. The Value of Neutrophil to Lymphocyte Ratio and Platelet to Lymphocyte Ratio for Detecting Early-onset Neonatal Sepsis. J Pediatr Hematol Oncol 2018; 40(4):e229-e32. doi: 10.1097/mph. 0000000000001059.

18. Dirican A, Kucukzeybek BB, Alacacioglu A, Kucukzeybek Y, Erten $C$, Varol $U$, et al. Do the derived neutrophil to lymphocyte ratio and the neutrophil to lymphocyte ratio predict prognosis in breast cancer? Int J Clin Oncol 2015; 20(1):70-81. doi: 10.1007/s10147-014-0672-8.

19. de Jager CP, Wever PC, Gemen EF, Kusters R, van Gageldonk-Lafeber AB, van der Poll $T$, et al. The neutrophillymphocyte count ratio in patients with communityacquired pneumonia. PLoS One 2012; 7(10):e46561. doi: 10.1371/journal.pone.0046561.

20. Wu Y, Chen $Y$, Yang $X$, Chen L, Yang $Y$. Neutrophil-tolymphocyte ratio (NLR) and platelet-to-lymphocyte ratio (PLR) were associated with disease activity in patients with systemic lupus erythematosus. Int Immunopharmacol 2016; 36:94-9. doi: 10.1016/j.intimp.2016.04.006.

21. Rifaioglu EN, Bülbül Şen B, Ekiz Ö, Cigdem Dogramaci A. Neutrophil to lymphocyte ratio in Behçet's disease as a marker of disease activity. Acta Dermatovenerol Alp Pannonica Adriat 2014; 23(4):65-7. 